\title{
Neutral mesons production measured with the ALICE experiment
}

\author{
Adam Matyja ${ }^{* \dagger}$ \\ The Henryk Niewodniczanski Institute of Nuclear Physics, Polish Academy of Sciences \\ ul. Radzikowskiego 152, 31-342 Kraków, Poland \\ E-mail: adam.tomasz.matyja@cern.ch
}

Inclusive neutral meson production spectra have been measured in a wide $p_{\mathrm{T}}$ range at mid-rapidity by the ALICE experiment in pp collisions from $\sqrt{s}=0.9$ to $13 \mathrm{TeV}, \mathrm{p}$-Pb collisions at $\sqrt{s_{\mathrm{NN}}}=$ 5.02 and $8.16 \mathrm{TeV}$, as well as $\mathrm{Pb}-\mathrm{Pb}$ collisions at $\sqrt{s_{\mathrm{NN}}}=2.76$ and $5.02 \mathrm{TeV}$. $\pi^{0}$ and $\eta$ mesons are reconstructed by their two photon decay channels using the invariant mass technique. The photons are detected with two complementary methods: photons converted to $e^{+} e^{-}$pairs in the central barrel detectors' material or directly registered in the ALICE electromagnetic calorimeters, EMCal and PHOS. An overview of the recent results from ALICE on mesons production in pp, $\mathrm{p}-\mathrm{Pb}$ and $\mathrm{Pb}-\mathrm{Pb}$ collisions is presented.

13th International Workshop in High pT Physics in the RHIC and LHC Era (High-pT2019)

19-22 March 2019

Knoxville, Tennessee, USA

\footnotetext{
* Speaker.

${ }^{\dagger}$ On behalf of the ALICE collaboration.
} 


\section{Introduction}

The ALICE experiment has been designed to study the Quark-Gluon Plasma (QGP) which is formed in heavy-ion (HI) collisions at the LHC. To disentangle ordinary nuclear effects from effects of a QGP one needs to refer to proton-proton or proton-nucleus collisions, which serve as a reference. The measurement of the differential production cross-section of neutral mesons is important for various reasons. At sufficiently large momentum, the calculation of the cross section can be factorized into short distance (perturbative) and long distance (non-perturbative) terms. Thus the measurement gives a chance to test NLO or NNLO pQCD calculations and to constrain parton distributions as well as fragmentation functions. Because the meson production is so well understood in pp collisions, neutral mesons can be used as precision probes also in HI collisions to study the QGP and possibly distinguish between different effects. The measurement of the neutral meson spectra constrains mechanisms of parton energy loss in dense systems via studies of the nuclear modification factor. In addition, looking into the cross-section ratio allows studying the $m_{\mathrm{T}}$ scaling, which is particularly important in the low- $p_{\mathrm{T}}$ regime.

\section{Neutral meson reconstruction}

Photons are detected in two ALICE electromagnetic calorimeters, EMCal and PHOS. They are also reconstructed by combining $e^{+} e^{-}$pairs produced when photons convert in the material of the central barrel detectors (called Photon Conversion Method). The $\pi^{0}$ and $\eta$ mesons are reconstructed via invariant mass technique of two photons using any of the three methods $\left(M_{\gamma \gamma}=\right.$ $\sqrt{2 E_{1} E_{2}(1-\cos \alpha)}$, where $E_{1}$ and $E_{2}$ are photon candidates energies and $\alpha$ is an opening angle between them) or via the shower shape of two overlapped electromagnetic cascades in EMCal in the case of the decay of a high energy $\pi^{0}$. The neutral meson differential production cross-section $E \frac{\mathrm{d}^{3} \sigma}{\mathrm{d} p^{3}}$ (or the invariant yield $E \frac{\mathrm{d}^{3} N}{\mathrm{~d} p^{3}}$, when the trigger cross-section is not known) can be written in the form, $E \frac{\mathrm{d}^{3} \sigma}{\mathrm{d} p^{3}}=\frac{1}{2 \pi p_{\mathrm{T}}} \frac{1}{L} \frac{1}{B R} \frac{1}{\varepsilon_{\text {eff }}} \frac{N_{\text {meson }}}{\Delta p_{\mathrm{T}} \Delta y}$, where the luminosity $L=N_{\mathrm{evt}} R_{\mathrm{trig}} / \sigma_{\mathrm{MB}}$ is expressed in terms of the number of events $N_{\text {evt }}$, the trigger rejection factor $R_{\text {trig }}$ and the minimum bias trigger cross-section $\sigma_{\mathrm{MB}}$, and $B R$ is the double photonic branching ratio, $\varepsilon_{\mathrm{eff}}$ is the efficiency for reconstructing the meson in the considered detector acceptance and $N_{\text {meson }}$ is the feed-down corrected neutral meson yield in the transverse momentum interval $\Delta p_{\mathrm{T}}$ and rapidity interval $\Delta y$. The spectra measured with these different techniques are in good agreement and are presented as a combined result, taking into account the correlated uncertainties for the combination using the BLUE method [3].

\section{Neutral mesons in $\mathrm{pp}, \mathrm{p}-\mathrm{Pb}$ and $\mathrm{Pb}-\mathrm{Pb}$ collisions}

Neutral meson spectra. ALICE has published the $\pi^{0}$ meson differential production crosssection in pp collisions at $\sqrt{s}=0.9[4,5], 2.76[6,7], 5[8,9], 7[4]$ and $8[10] \mathrm{TeV}$ as well as the invariant yield in $\mathrm{p}-\mathrm{Pb}$ collisions at $\sqrt{s_{\mathrm{NN}}}=5.02 \mathrm{TeV}[11,12]$ and $\mathrm{Pb}-\mathrm{Pb}$ collisions at $\sqrt{s_{\mathrm{NN}}}=2.76[6,13]$ and $5.02 \mathrm{TeV}[8]$ in several centrality classes. Figure 1 shows preliminary high precision $\pi^{0}$ meson production spectra in pp (left) and $\mathrm{Pb}-\mathrm{Pb}$ (right) at $\sqrt{s_{\mathrm{NN}}}=5.02 \mathrm{TeV}$ compared to NLO pQCD and various model predictions. The pp spectra are reasonably described by the NLO pQCD calculations, where agreement is within 20-40\% depending on $p_{\mathrm{T}}$ and predictions with a 

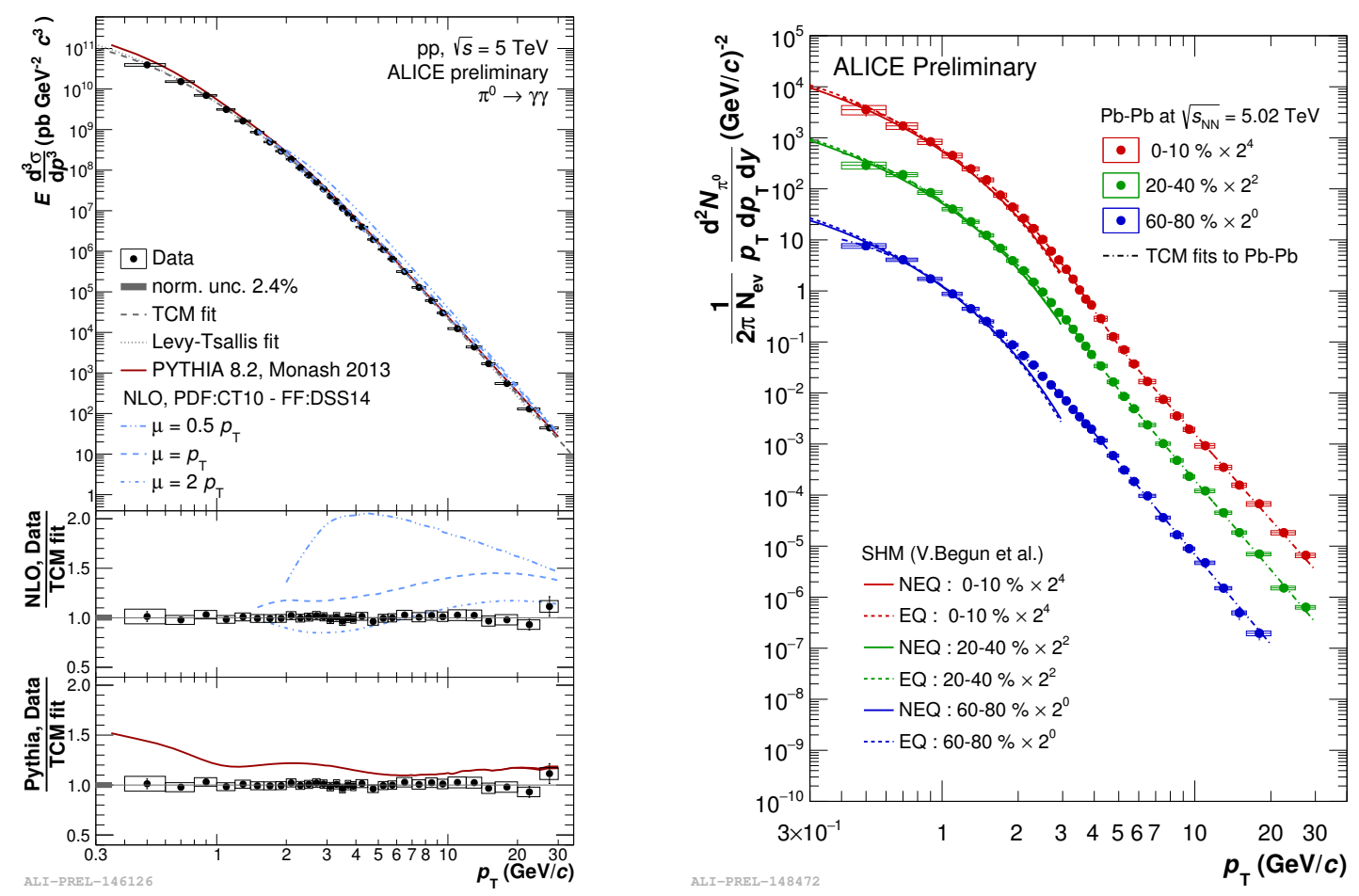

Figure 1: Left: The $\pi^{0}$ meson spectrum versus $p_{\mathrm{T}}$ in pp collisions at $\sqrt{s}=5.02 \mathrm{TeV}$ is shown in top panel. Result is compared to the PYTHIA 8 Monte Carlo [14] generator (red solid curve) and NLO pQCD predictions for three scales (blue curves). Corresponding ratios are shown in bottom panels. Right: The $\pi^{0}$ meson spectra for $0-10 \%$ (red dots), $20-40 \%$ (green dots) and $60-80 \%$ (blue dots) $\mathrm{Pb}-\mathrm{Pb}$ collsions at $\sqrt{s_{\mathrm{NN}}}=5.02 \mathrm{TeV}$ compared to EQ and NEQ versions of SHM [15] model.

higher factorization and renormalization scale describe pp data better. The PYTHIA 8 generator (Monash 2013 tune [14]) overpredicts pp data in the whole $p_{\mathrm{T}}$ range up to $40 \%$. For $p_{\mathrm{T}}<2 \mathrm{GeV} / c$, the $\mathrm{Pb}-\mathrm{Pb}$ spectra in centrality classes are well reproduced by the hydro inspired SHM [15] model.

$\eta / \pi^{0}$ cross-section ratio. ALICE has measured the $\eta / \pi^{0}$ cross-section ratios for systems and energies where the $\eta$ meson spectrum was measured. The $\eta / \pi^{0}$ ratio in pp collisions [10] at $\sqrt{s}=2.76,7$ and $8 \mathrm{TeV}$, in $\mathrm{p}-\mathrm{Pb}$ collisions at $\sqrt{s_{\mathrm{NN}}}=5.02 \mathrm{TeV}$ and in the most central $\mathrm{Pb}-\mathrm{Pb}$ collisions at $\sqrt{s_{\mathrm{NN}}}=2.76 \mathrm{TeV}[13]$ is shown in Fig. 2. The $\eta / \pi^{0}$ ratio grows with increasing $p_{\mathrm{T}}$ and then levels off at $\sim 0.5$ above $p_{\mathrm{T}} \sim 3 \mathrm{GeV} / c$. Results show the universal character among collision energies and systems, and agree within uncertainties with the world data [4]. The significant (above $6 \sigma$ for pp collisions at $\sqrt{s}=8 \mathrm{TeV}$ ) deviation from $m_{\mathrm{T}}$ scaling prediction at low- $p_{\mathrm{T}}$ is visible in pp collsions as seen also for other mesons at LHC energies [16]. The origin is most likely from additional contributions to light mesons from heavier resonance decays. A similar behavior is seen in $\mathrm{p}-\mathrm{Pb}$ and $\mathrm{Pb}-\mathrm{Pb}$ collisions. An additional effect coming from the onset of flow may result in an enhancement between $p_{\mathrm{T}} \approx 1-3 \mathrm{GeV} / c$ as in the $K^{ \pm} / \pi^{ \pm}$ratio for the most central p-Pb collisions. There is no clear onset seen yet in the $\eta / \pi^{0}$ ratio due to the larger uncertainties. However, a hint of enhancement is seen in $\mathrm{Pb}-\mathrm{Pb}$ collisions, which are well reproduced by models including flow (SHM [15] or EPOS [20]).

Nuclear modification factor. The nuclear modification factor $R_{\mathrm{AA}}$ (or $Q_{\mathrm{pA}}$ for $\mathrm{p}-\mathrm{Pb}$ collisions 


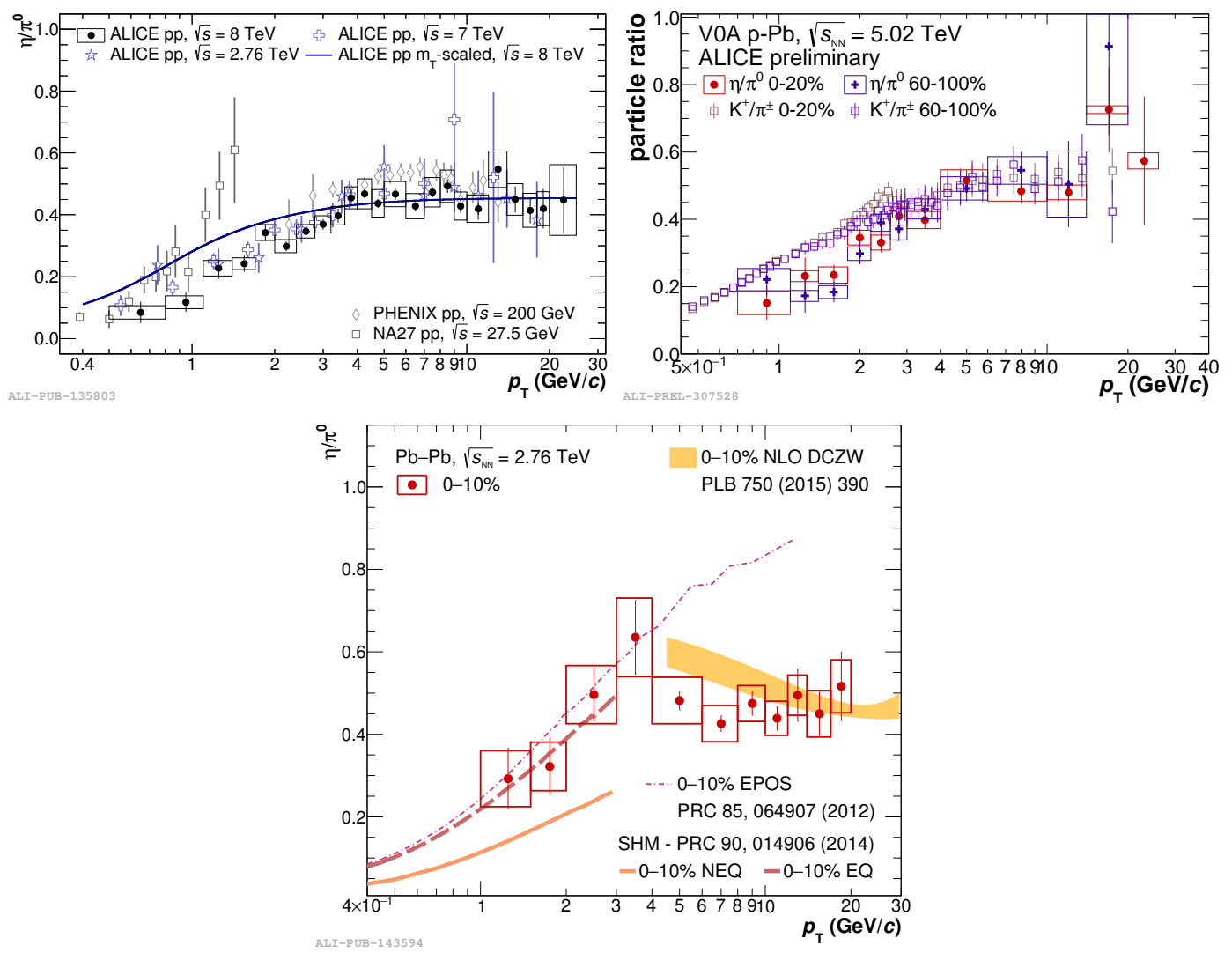

Figure 2: The $\eta / \pi^{0}$ cross-section ratio in pp [10] at $\sqrt{s}=2.76,7$ and $8 \mathrm{TeV}$ compared to $m_{\mathrm{T}}$ scaling predictions and lower energy experiments $[17,18]$ (top left), $\mathrm{p}-\mathrm{Pb}$ (top right) for most central and peripheral collisions compared to $K^{ \pm} / \pi^{ \pm}$ratios at $\sqrt{s_{\mathrm{NN}}}=5.02 \mathrm{TeV}$ and $\mathrm{Pb}-\mathrm{Pb}$ (bottom) collisions at $\sqrt{s_{\mathrm{NN}}}=2.76 \mathrm{TeV}$ [13] compared to NLO pQCD calculations [19] or SHM [15] and EPOS [20] models.

to differenciate between different centrality estimators) quantifies the effect in nuclear collisions in comparison to $p$ collisions, $R_{\mathrm{AA}}\left(p_{\mathrm{T}}\right)=\frac{\mathrm{d}^{2} N /\left.\mathrm{d} p_{\mathrm{T}} \mathrm{d} y\right|_{\mathrm{AA}}}{\left\langle T_{\mathrm{AA}}>\times \mathrm{d}^{2} \sigma /\left.\mathrm{d} p_{\mathrm{T}} \mathrm{d} y\right|_{\mathrm{pp}}\right.}$, where the numerator represents the differential yield in AA collisions, while the denominator is the differential cross-section in $\mathrm{pp}$ collisions scaled by $\left\langle T_{\mathrm{AA}}\right\rangle$, the nuclear overlap factor. There are several methods to determine centrality in $\mathrm{p}-\mathrm{Pb}$ collisions [21] due to large fluctuations and possible correlations between the multiplicity estimator and the measured spectrum. The CL1 estimator is based on tracklets in the pseudo-rapidity range $|\eta|<1.4$, the V0A estimator covers the $2<\eta<5.1$ range and the ZNA measures neutral energy on the $\mathrm{Pb}$-side in the Zero Degree Calorimeter in the $|\eta|>8.7$ range. The ZNA estimator is the least biased [21]. The apparent "suppression" of the yield for the most peripheral class for CL1 and V0A estimators in p-Pb collisions can lead to incorrect conclusions. One should be very cautious with interpretation of the results.

The neutral pion $Q_{\mathrm{pA}}$ for different centrality classes measured with different estimators in $\mathrm{p}-\mathrm{Pb}$ collisions at $\sqrt{s_{\mathrm{NN}}}=5.02 \mathrm{TeV}$ are shown in Fig. 3. There is no centrality dependence for ZNA (the largest rapidity gap), while V0A and CL1 show strong and similar dependence (smaller rapidity gap). The $Q_{\mathrm{pA}}$ for $\pi^{0}, \eta$ and $D^{0}$ meson as well as for charged hadrons in the most central $\mathrm{p}-\mathrm{Pb}$ collisions at $\sqrt{s_{\mathrm{NN}}}=5.02 \mathrm{TeV}$ are shown in the top left plot in Fig. 4. Agreement within 

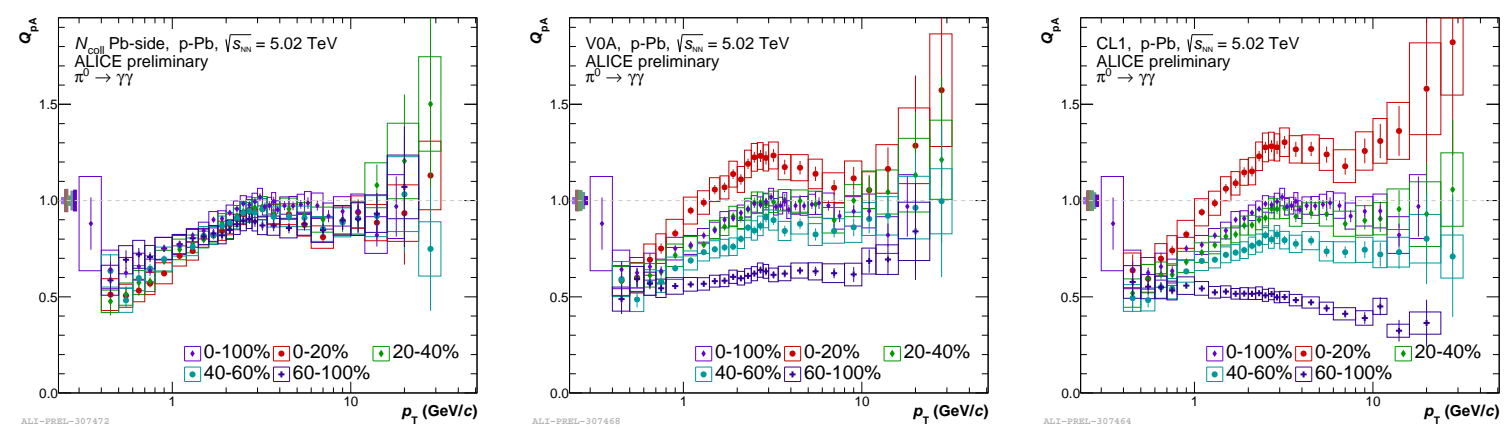

Figure 3: $Q_{\mathrm{pA}}$ vs $p_{\mathrm{T}}$ in p-Pb collisions at $\sqrt{s_{\mathrm{NN}}}=5.02 \mathrm{TeV}$ in centrality classes measured via ZNA (left), VOA (middle) and CL1 (right) estimators.

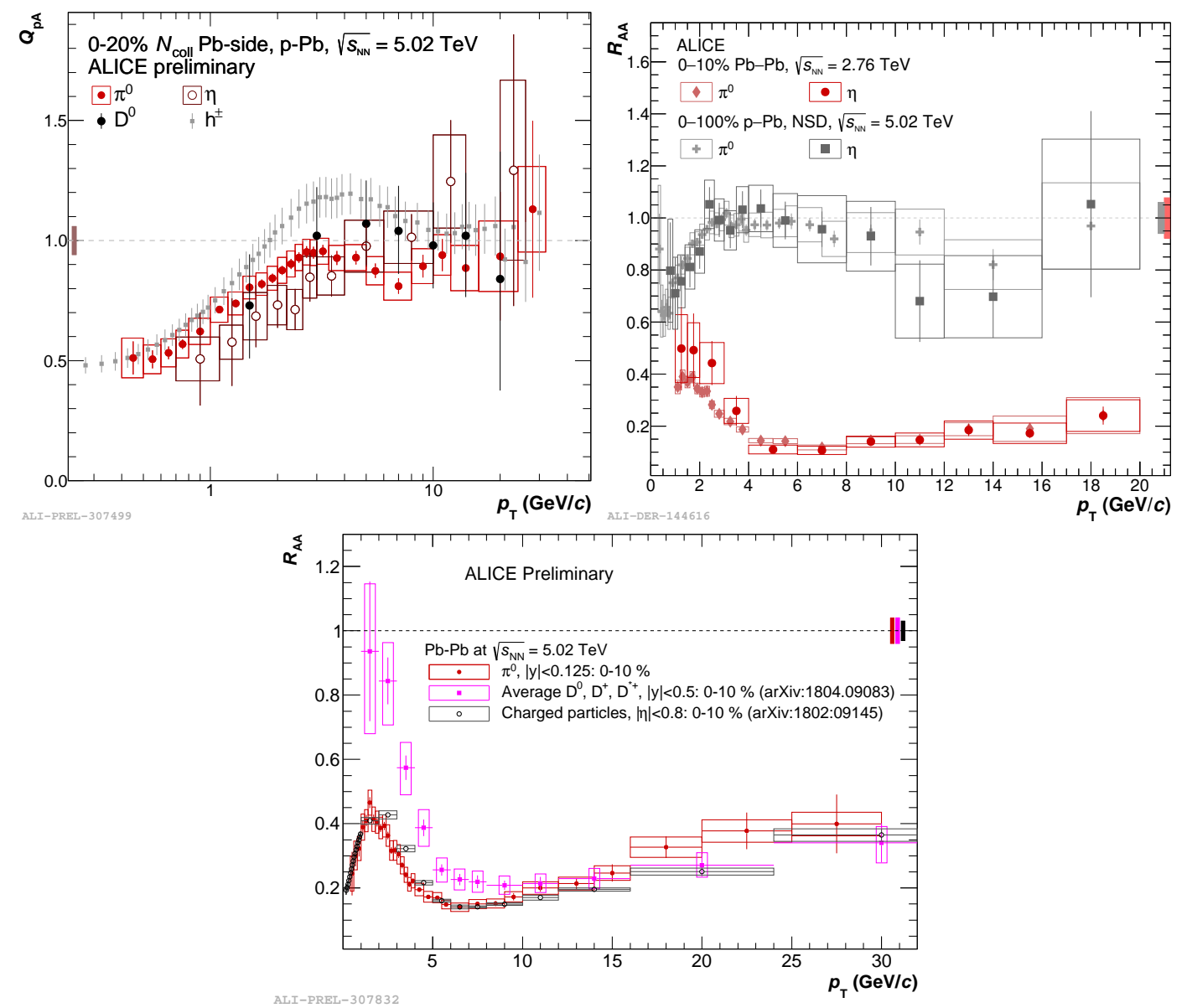

Figure 4: The nuclear modification factor versus $p_{\mathrm{T}}$. Top left: The $\pi^{0}$ (red dots) and $\eta$ (open brown circles) mesons in the most central $0-20 \% \mathrm{p}-\mathrm{Pb}$ collisions at $\sqrt{s_{\mathrm{NN}}}=5.02 \mathrm{TeV}$ are compared to charged hadrons (gray squares) and $D^{0}$ mesons (black dots). Top right: The $\pi^{0}$ (diamonds) and $\eta$ (dots) mesons in $0-10 \%$ most central $\mathrm{Pb}-\mathrm{Pb}$ collisions at $\sqrt{s_{\mathrm{NN}}}=2.76$ TeVcompared to non-single diffractive $\mathrm{p}-\mathrm{Pb}$ collsions at $\sqrt{s_{\mathrm{NN}}}=5.02 \mathrm{TeV}$ for the $\pi^{0}$ (crosses) and $\eta$ (squares) mesons. Bottom: The $\pi^{0}$ (red dots) in $\mathrm{Pb}-\mathrm{Pb}$ collisions at $\sqrt{s_{\mathrm{NN}}}=5.02 \mathrm{TeV}$ are compared to average D mesons (pink squares) and charged particles (open black circles) at the same energy and centrality. 
uncertainties is visible for each identified meson $Q_{\mathrm{pA}}$. The bump around $p_{\mathrm{T}} \sim 3 \mathrm{GeV} / c$ visible for unidentified hadrons is probably caused by protons [21]. The $R_{\mathrm{AA}}$ for $\pi^{0}$ and $\eta$ mesons in $0-10 \%$ central $\mathrm{Pb}-\mathrm{Pb}$ collisions at $\sqrt{s_{\mathrm{NN}}}=2.76 \mathrm{TeV}$ are compared to $\mathrm{MB}(0-100 \%) \mathrm{p}-\mathrm{Pb}$ collisions at $\sqrt{s_{\mathrm{NN}}}=5.02 \mathrm{TeV}$ in the top right plot of Fig. 4. No suppression in $\mathrm{p}-\mathrm{Pb}$ collisions and strong suppression of meson yield in $\mathrm{Pb}-\mathrm{Pb}$ collisions indicates that this suppression is due to a final state effect. The similar suppression pattern above $p_{\mathrm{T}}=4 \mathrm{GeV} / c$ for both mesons indicates that suppression occurs at the partonic level. The suppression of different particle species in $\mathrm{Pb}-\mathrm{Pb}$ collisions at $\sqrt{s_{\mathrm{NN}}}=5.02 \mathrm{TeV}$ is shown in the bottom plot of Fig. 4. The similar suppression above $p_{\mathrm{T}} \approx 10 \mathrm{GeV} / c$ is visible for all the considered particles. Below this value $\pi^{0}$ and charged particles show the same amount of suppression while D-mesons show less suppression. Different masses, flow, quark content or recombination pattern can be the potential source of this hierarchy, which still remains to be further disentangled.

\section{Summary}

The ALICE experiment has measured $\pi^{0}$ and $\eta$ meson production spectra, their ratios and nuclear modification factors in $\mathrm{pp}, \mathrm{p}-\mathrm{Pb}$ and $\mathrm{Pb}-\mathrm{Pb}$ collisions at LHC energies. A theoretical description of measured observables is far from fully satisfactory. However, calculations and MC generator predictions agree with measured quantities in some $p_{\mathrm{T}}$ regions well. There is still a lot of room for improvements. The observed $m_{\mathrm{T}}$ scaling violation at low $p_{\mathrm{T}}$ in every collision system and a hint of flow in $\mathrm{Pb}-\mathrm{Pb}$ collisions indicate the need for more precise measurements. The nuclear modification factor in $\mathrm{p}-\mathrm{Pb}$ collisions is very sensitive to the used centrality estimator. One needs to carrefully interpret the results. There is no difference within uncertainties between light neutral mesons and $\mathrm{D}$ mesons $Q_{\mathrm{pA}}$ in $\mathrm{p}-\mathrm{Pb}$ collisions while a hierarchy $\left(R_{\mathrm{AA}}(D)<R_{\mathrm{AA}}\left(\pi^{0}\right)\right)$ is seen at $p_{\mathrm{T}}<10 \mathrm{GeV} / c$ in $\mathrm{Pb}-\mathrm{Pb}$ collisions at $\sqrt{s_{\mathrm{NN}}}=5.02 \mathrm{TeV}$.

\section{Acknowledgments}

We would like to thank the Ministry of Science and Higher Education and the National Science Centre, Poland for the supporting grants.

\section{References}

[1] K. Aamodt et al. (ALICE Collaboration), The ALICE experiment at the CERN LHC, JINST 3 (2008) S08002.

[2] L. Evans et al., LHC Machine, JINST 3 (2008) S08001.

[3] L. Lyons, D. Gibaut, P. Clifford, How to combine correlated estimates of a single physical quantity, Nucl. Instrum.Methods A 270 (1988) 110.

[4] B. Abelev et al. (ALICE Collaboration), Neutral pion and $\eta$ meson production in proton-proton collisions at $\sqrt{s}=0.9 \mathrm{TeV}$ and $\sqrt{s}=7 \mathrm{TeV}$, Phys. Lett. B717 (2012) 162 [arXiv: 1205.5724 ].

[5] K. Reygers (ALICE Collaboration), Production of Neutral Pions and Eta-mesons in pp Collisions Measured with ALICE, in proceedings of 22nd International Conference on Ultra-Relativistic Nucleus-Nucleus (Quark Matter 2011), J. Phys. G38 (2011) 124076 [arXiv: 1106.5932$].$ 
[6] B. Abelev et al. (ALICE Collaboration), Neutral pion production at midrapidity in pp and $\mathrm{Pb}-\mathrm{Pb}$ collisions at $\sqrt{s_{\mathrm{NN}}}=2.76$ TeV, Eur. Phys. J. C74 (2014) 3108 [arXiv: 1405.3794$]$.

[7] S. Acharya et al. (ALICE Collaboration), Production of $\pi^{0}$ and $\eta$ mesons up to high transverse momentum in pp collisions at 2.76 TeV, Eur. Phys. J. C77 (2017) 339. [arXiv:1702 . 00917 ].

[8] D. Sekihata (ALICE Collaboration), Energy and system dependence of nuclear modification factors of inclusive charged particles and identified light hadrons measured in $\mathrm{p}-\mathrm{Pb}, \mathrm{Xe}-\mathrm{Xe}$ and $\mathrm{Pb}-\mathrm{Pb}$ collisions with ALICE, in proceedings of Quark Matter 2018, [arXiv: 1807.11240] (2018).

[9] A. Matyja (ALICE Collaboration), Neutral meson and photon production at the LHC measured with the ALICE experiment, in proceedings of 21st High-Energy Physics International Conference in Quantum Chromodynamics (QCD 18), Nucl. Part. Phys. Proc. 300-302 (2018) 99-106.

[10] S. Acharya et al. (ALICE Collaboration), $\pi^{0}$ and $\eta$ meson production in proton-proton collisions at $\sqrt{s}=8$ TeV, Eur. Phys. J. C78 (2018) 263 [arXiv: 1708 . 08745].

[11] S. Acharya et al. (ALICE Collaboration), Neutral pion and $\eta$ meson production in $p$-Pb collisions at $\sqrt{s_{\mathrm{NN}}}=5.02$ TeV, Eur. Phys. J. C78 (2018) 624 [arXiv: 1801.07051$]$.

[12] D. Peresunko (ALICE Collaboration), Overview of neutral-meson production in $p p$, $p$ - $A$ and $A-A$ collisions at the LHC measured by ALICE, in proceedings of International Conference on Hard and Electromagnetic Probes of High-Energy Nuclear Collisions, PoS(HardProbes2018) (2018) 073.

[13] S. Acharya et al. (ALICE Collaboration), Neutral pion and $\eta$ meson production at mid-rapidity in Pb-Pb collisions at $\sqrt{s_{\mathrm{NN}}}=2.76$ TeV, Phys. Rev. C98 (2018) 044901 [arXiv: 1803.05490 ].

[14] P. Skands, S. Carrazza, J. Rojo, Tuning PYTHIA 8.1: the Monash Tune, Eur. Phys. J. C74 (2014) 3024 [arXiv: 1404.5630$]$.

[15] V. Begun, W. Florkowski, M. Rybczynski, Explanation of hadron transverse-momentum spectra in heavy-ion collisions at $\sqrt{s_{\mathrm{NN}}}=2.76 \mathrm{TeV}$ within chemical non-equilibrium statistical hadronization model, Phys. Rev. C90 (2014) 014906 [arXiv: 1312 . 1487 ].

[16] L. Altenkämper, F. Bock, C. Loizides, N. Schmidt, Applicability of transverse mass scaling in hadronic collisions at energies available at the CERN Large Hadron Collider, Phys. Rev. $\mathbf{C 9 6}$ (2017) 064907 [arXiv:1710.01933].

[17] A. Adare et al. (PHENIX Collaboration), Cross section and double helicity asymmetry for eta mesons and their comparison to neutral pion production in $p+p$ collisions at $\sqrt{s}=200 \mathrm{GeV}$, Phys. Rev. D83 (2011) 032001 [arXiv:1009.6224].

[18] M. Aguilar-Benitez et al. (NA27 Collaboration), Inclusive particle production in $400 \mathrm{GeV/c}$ pp-interactions, Z. Phys. C50 (1991) 405-426.

[19] W. Dai, X.-F. Chen, B.-W. Zhang, and E. Wang, $\eta$ meson production of high-energy nuclear collisions at NLO, Phys. Lett. B750 (2015) 390-395 [arXiv: 1506.00838 ].

[20] K. Werner, I. Karpenko, M. Bleicher, T. Pierog, and S. Porteboeuf-Houssais, Jets, bulk matter, and their interaction in heavy-ion collisions at several TeV, Phys. Rev. C85 (2012) 064907 [arXiv:1203.5704].

[21] J. Adam et al. (ALICE Collaboration), Centrality dependence of particle production in $p-P b$ collisions at $\sqrt{s_{\mathrm{NN}}}=5.02$ TeV, Phys. Rev. C91 (2015) 064905 [arXiv:1412.6828]. 